Rapid Reviews COVID-19

\title{
Review 1: "Comparison of
} infection control strategies to reduce COVID-19 outbreaks in homeless shelters in the United States: a simulation study"

\section{Gregory Karelas ${ }^{1}$}

${ }^{1}$ Columbia University Mailman School of Public Health, Health Policy and Management, USA

Published on: Nov 06, 2020

DOI: $10.1162 / 2 \mathrm{e} 3983 f 5 . a c 878 f 41$

License: Creative Commons Attribution 4.0 International License (CC-BY 4.0). 


\section{$\underline{\text { RR:C19 Evidence Scale rating by reviewer: }}$}

- Reliable. The main study claims are generally justified by its methods and data. The results and conclusions are likely to be similar to the hypothetical ideal study. There are some minor caveats or limitations, but they would/do not change the major claims of the study. The study provides sufficient strength of evidence on its own that its main claims should be considered actionable, with some room for future revision.

$* * * * * * * * * * * * * * * * * * * * * * * * * * * * * * * * * * * * * * *$

\section{Review:}

I would recommend this preprint for publication with minor revisions.

This paper is interesting and novel. It could have great value to people directing-or receiving services from-homeless shelters and other settings of congregate living or high infection risk. Its design and methods are well-conceived, drawing data from shelters and cities of high relevance and predictive value. The article is also clearly written, succinctly detailing a model and findings with many components.

I would consider this paper's strength of evidence to be "Reliable," justified by its methods and data, and similar to the hypothetical ideal study. The manuscript clearly details any study limitations-none of which change its major claims.

The manuscript articulates the effectiveness of various approaches to infection control in settings of congregate living among people at high risk of infection. Its findings contribute to broader understandings of infection control and research. It cites the current literature extensively and stands to impact the implementation of policies on facility and city levels. The study's data are clear. And its focus on populations affected by homelessness is a step toward equity and social justice.

Some thoughts on how to improve this manuscript would include:

- Interventions: Articulate why this model chose its six interventions for simulation. The paper clearly states that "informal consultation" with public health experts contributed to their selection. More detail regarding the vetting process for these interventions, and why these six were ultimately selected versus other, fewer or more interventions, would be useful. 
- Implications: Consider detailing how the study's findings could be valuable to settings beyond homeless shelters. Articulation of how the simulation results could extend to other spaces would fit well in the abstract and conclusion. This should also include clarity regarding which types of spaces could be considered, thus mitigating the chance of misinterpretation and erroneously applied findings.

- Conclusions: More closely align the study's conclusions with its goal. In the abstract and introduction, the paper articulates the goal of finding the "most effective infection control strategy" to reduce outbreaks. Its conclusions recommend combined interventions for protecting people experiencing homelessness; but it does so based on data that indicate those interventions would only avert outbreaks in low-risk shelters. The paper does mention that strategies external to its analysis-eg: reduced living density, spaced bedding - could be beneficial in high-risk settings. Conclusions that specify recommendations for the "most effective infection control strategies" in both high- and low-risk shelters would strengthen its impact. 